\title{
Biophysics of Impact Injury to the Chest and Abdomen
}

\section{G J Cooper}

\section{BSc, PhD}

Medical Division, Chemical Defence Establishment, Porton, Salisbury, Wiltshire.

Honorary Senior Lecturer in Trauma Science, Department of Military Surgery, Royal Army Medical College, Millbank, London.

\section{DE M Taylor}

MB, ChB, FRCS, FRSE, TD

Professor, Department of Applied Physiology and Surgical Sciences,

Royal College of Surgeons of England, 35-43 Lincoln's Inn Fields, London.

SUMMARY: The physical mechanism of blunt impact injuries to thoracic and abdominal viscera is often conveniently described simply in terms of "crush" - this is an over-simplification. Any impact to the torso does result in the rapid displacement of the body wall which may lacerate and contuse underlying viscera, but this simple explanation does not account for pathology at sites some distance from the contact point and does not adequately describe the dependence of the severity and location of injury upon the rate of energy transfer. Quite minor displacements of the body wall may produce serious injury if the body wall velocity is high. The motion of the body wall generates waves that propagate within the body and transfer energy to internal sites. The nature and properties of these waves are discussed in simple terms and the role of waves in the production of the characteristic injuries resulting from impact to the torso is presented.

\section{Drummer boys Lew and Jakin contemplate their fitness} for battle:

"Are you medical fit, Piggy?" said Jakin, digging Lew in the ribs with force.

"Yus," said Lew with an oath. "The Doctor says your 'eart's weak through smokin' on an empty stummick. Throw a chest an' I'll try yer."

Jakin threw out his chest, which Lew smote with all his might. Jakin turned very pale, gasped, crowed, screwed up his eyes and said - "That's all right."

"You'll do," said Lew. I've 'eard o' men dying when you 'it'em fair on the breastbone."

The Drums of the Fore and Aft (R Kipling)

\section{Introduction}

Aristotle is credited with one of the first observations on the result of non-penetrating or blunt impact injury when he recognised that a blow to the belly of a deer could produce intestinal perforation ${ }^{1}$. Over the subsequent centuries the aetiology of blunt or nonpenetrating (NP) impact injury remained largely accidents with horses, horse drawn vehicles, falls and affrays, but in more modern times the principal cause of non-penetrating injury to the torso in the civilian context is the road traffic accident.

The association of NP impact injuries and motor vehicles extends into the military population of course but in addition, the soldier in combat or engaged in internal security duties may be subjected to the additional potential NP impact hazards of exposure to blast overpressure or the deformation of flexible body armour produced by retardation of bullets.

The principal cause of NP injury remains road traffic accidents and over the years there has been progress in the steps taken to mitigate the exchange of energy between the human and the vehicle. The most obvious step has been the enactment of seat belt legislation, but rather more subtle and less obvious changes have occurred in the design of motor vehicles - the profusion of plastic, foam and rounded surfaces in the interiors and exteriors of modern motor vehicles is testament to the aim of energy absorption and the distribution of contact forces. An understanding of the biophysical principles of impact injury to the human body and the aetiology of the resulting pathology is necessary if advances are to be 8 made in the prevention or mitigation of injury ${ }^{2}$.

Lew's observations described above on the potentiale result of the application of force to the anterior thoracic wall will arouse in the pathologist thoughts of arrhythmias either in the presence or absence of cardiac contusion, cardiac rupture or aortic rupture. Although it is perhaps reasonable to predict that a blow to a site such as the sternum may deform the chest and produce contusion or even laceration to the right ventricle immediately under the impact site, it is a little more difficult to understand why impacts at this site may also result in contusion of the posterior surface of the heart or rupture of the proximal descending thoracic aorta deep within the thorax.

The injuries described above that may arise from an impact to the anterior chest illustrate a general classification of location of internal injury with respect to the impact site. Injuries may be classed as either direct or indirect:

DIRECT are those injuries underlying or immediately adjacent to the deformation of the body wall.

INDIRECT occur remote from the site of body wall displacement. 
The purpose of this paper is to briefly review the biomechanical principles of impact injury to the torso and to identify the physical phenomena that may be responsible for both direct and indirect injury at thoracic and abdominal sites.

\section{Mechanisms of Injury}

If a blunt projectile is propelled towards the body wall or conversely the mobile body strikes a stationery object, a load or force is applied to the body wall. The thorax in particular may appear to be a relatively stiff structure when pushed 'statically' by hand, but under the magnitude of the loads applied by impacts, the chest wall may undergo considerable deformation at the impact site and within the immediate area. Even the humble cricket ball may apply average loads of up to $1 / 4$ tonne to the chest - this bald statement must be qualified by stating that the load is only applied for 2-3 milliseconds.

\section{Motion of the body wall}

The rapid inward displacement of the body wall is the primary mechanical response of the body wall to the loading and is the principal factor responsible for the transfer of energy into the body and the production of internal injury (Fig 1). It will be shown later that different features of this motion (the maximum displacement produced and the velocity attained in the early stages of the motion) may be responsible for different classes of injury; without some degree of motion, there would be no transfer of energy internally.

Inspection of high-speed films of experimental impacts of non-penetrating projectiles upon the torsos of anaesthetised pigs reveals that subsequent to the rapid deformation of the body wall at the contact site, the projectile has very little rebound velocity and the body wall returns very slowly to its original shape. The projectile simply distorts the body wall and falls awaythis denotes that the thoracic or abdominal wall is not acting solely like a simple spring being compressed and then releasing this energy back to the projectile. The lack of significant rebound velocity shows that the body is highly damped or "viscous"; the significance of this observation is that for a system having an elastic and viscous behaviour (viscoclastic), the displacement upon impact loading is rate dependant. The faster that a viscoelastic material is deformed, the more resistance it offers (it becomes stiffer). This behaviour is broadly similar to that of a car suspension; a slow push will depress the suspension but the shock absorber stiffens the suspension to severe blows during driving.

The type and severity of internal injury is dependant not only upon the magnitude of the distortion of the body wall, but also upon the rate at which distortion occurs.

The torso can withstand quite severe compressions resulting in only minor injury (even up to a $50 \%$ reduction in body dimcnsions in the dircetion of the applied force) if the rate of the distortion is slow (slow in this context is attaining pcak compression in grcater than about 20-50 milliscconds). Converscly, high velocily distortions occurring over short periods of time (less than 5 milliseconds) may produce serious internal injury even with body wall distortions of only $3-4 \mathrm{~cm}^{3}$ (Fig 2)

The sensitivity of internal injury upon the rate of body wall distortion results from i) the mechanical failure (injury) in most materials, including tissues, being dependant upon the rate of strain, and ii) the propagation of different types of waves into the thorax.
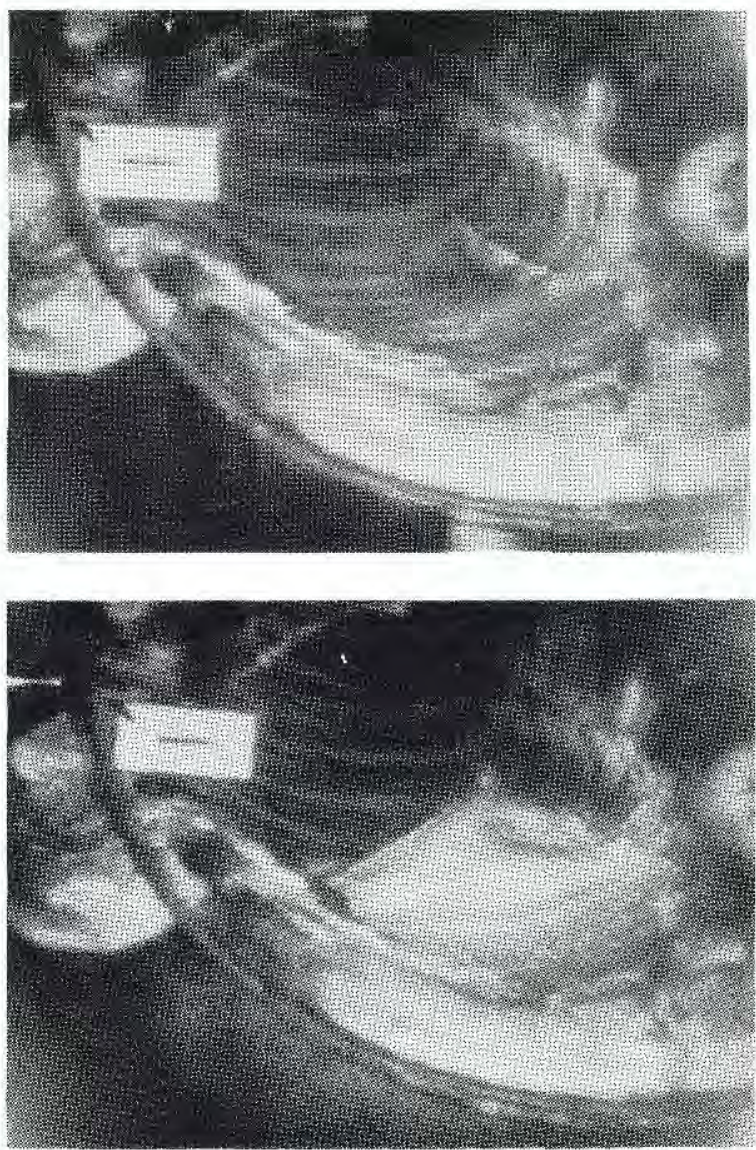

Fig I. Internal thoracic wall distortion produced by impact to the lateral thoracic wall by a $37 \mathrm{~mm}$ diameter, $0.14 \mathrm{~kg}$ projectile at $\mathbf{4 0} \mathrm{m} / \mathrm{s}$. (a) shows a pre-impact still acquired from high-speed cine film taken with the camera viewing cranially into the eviscerated thorax of a pig. The lateral thoracic wall is at the bottom of the image and the rib border is in the foreground. (b) is taken 3 milliseconds after contact and shows chest displacement with rib fracture and the strain induced in the intercostal muscles. Evisceration of the thorax does not significantly modify the response of the thoracic wall to impact. 

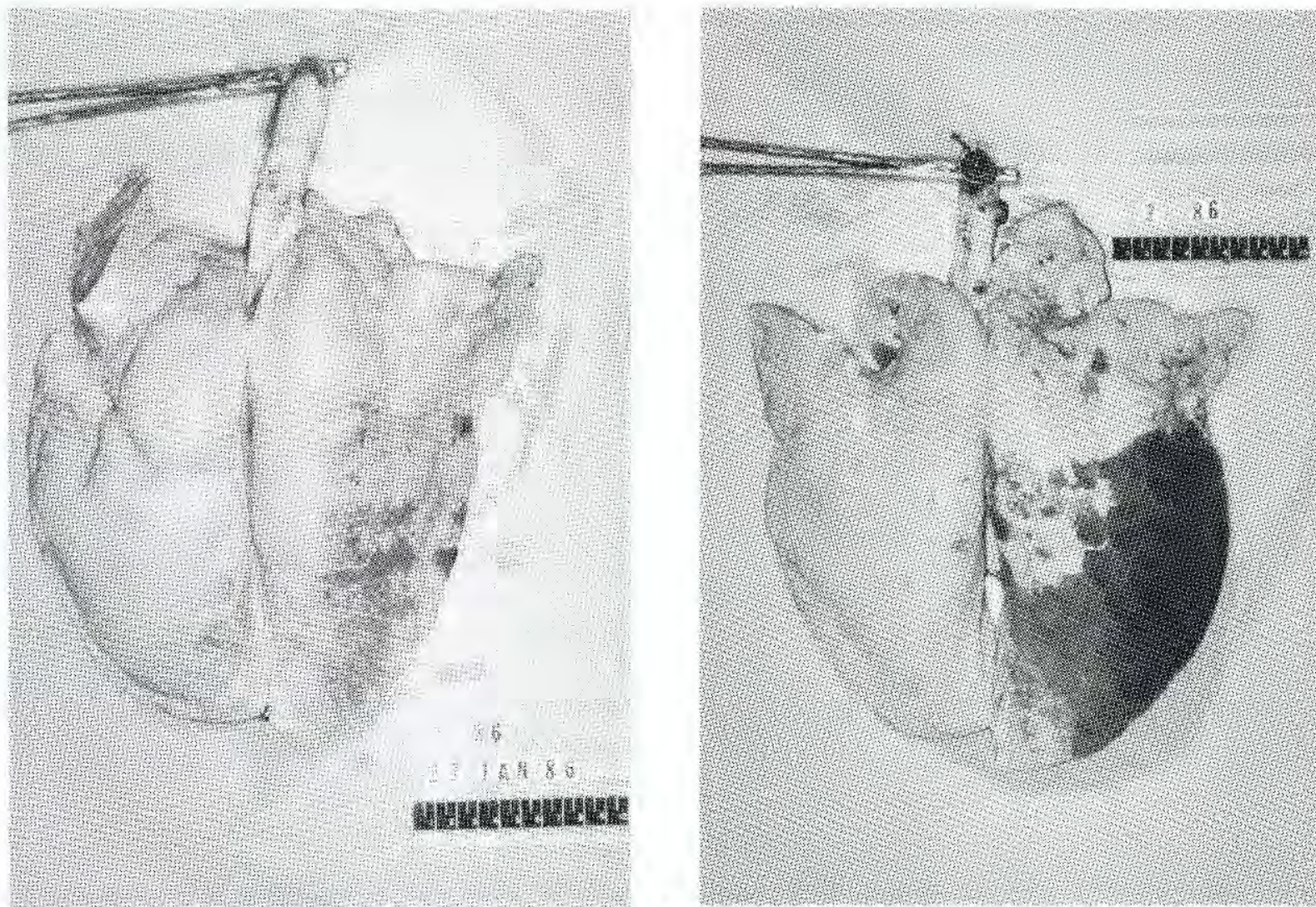

Fig 2. The gross displacement of the body wall is not the sole determinant of injury severity. (a) shows minor contusion injury to the lungs of a pig following severe chest compression of the right lateral thorax. A peak displacement of $10 \mathrm{~cm}$ was produced within 17 milliseconds. Compare this to the contusions in (b) produced by a distortion of only $4 \mathrm{~cm}$. However, the duration of the impact in (b) was only 1.2 milliseconds. Stress waves produced by the higher velocity of the body wall in (b) probably account for the more serious injury.

The different wave types have different capacities to injure the internal organs and an understanding of these wave effects may help to explain not only the type and severity of pathology produced in particular organs by a non-penetrating impact, but also the aetiology of direct and of indirect injury.

\section{Waves and soft tissue injury}

Four mechanisms of internal injury may be identified, three of which involve the propagation of waves through the body.

Stress waves - Iongitudinal pressure waves (rather like sound) that travel at or slightly faster than the velocity of sound in the tissue. They differ from sound by having very high amplitude.

Shock waves - waves of high pressure characterised by an effectively instantaneous wavefront propagated through the underlying tissue at a velocity faster than the velocity of sound in the tissue.
A shock wave can be considered to be a special form of a stress wave.

Shear waves - transverse waves of long duration and very low velocity producing gross distortions of tissues and organs (waves rather like the pulse produced by the whipping of a loose rope).

Crush injury - that is not rate dependent but simply a consequence of applying effectively static intense loads to tissue.

The relative contribution of different wave types to the total response to an impact will depend upon the rate of energy application at the impact site and the degree of compression produced. 'Fast' impacts such as blast will tend to excite the high frequency compression waves, whereas 'slow' impacts typified by retardation of the mobile body by an obstacle will tend to provoke low frequency shear waves with large, slow displacements. Impact by NP projectiles may span these frequencies. 
Stress waves. Stress (compression) waves in tissue (Fig 3) may result in very high local forces producing small but very rapid distortions of tissue (strain). In organs incorporating marked differences in material properties such as tissue and air (eg the lungs and bowel) there may be significant pressure differentials across rather delicate barriers such as alveoli during the

- propagation of the wave. Stress waves do not usually result in gross lacerations to tissues, their effects are largely concentrated at the microvascular level to produce extravasation of blood. Most pulmonary contusions and bowel contusions are probably produced by stress waves and in general, stress wave induced injuries mainly affect air containing organs or any other system having marked discontinuities in material properties. It is at these interfaces that energy may be released (eg lithotripsy to disrupt 'hard' kidney stone surrounded by 'soft' tissue). Percussion of the chest uses stress waves (of very low amplitude) to assess the degree of consolidation of air-containing organs.

Stress waves have two characteristics that may result in a high incidence of indirect injuries.

1. they will reflect from an interface such as the mediastinum or the walls of large airways resulting in at least a doubling of the pressure at the surface.

2. subsequent to reflection, they may interact and reinforce to produce high localised pressures within the body away from the site of generation, the body wall.

This feature is illustrated in Figure 4 where a wave generated at the pleural surface of a lung by a localised impact may be amplified by reflection and reinforcement to produce a high pressure environment in the body of the lung ${ }^{4}$. In animals subjected to localised very high

TRIAL 1

RIGHT MAIN BRONCHUS

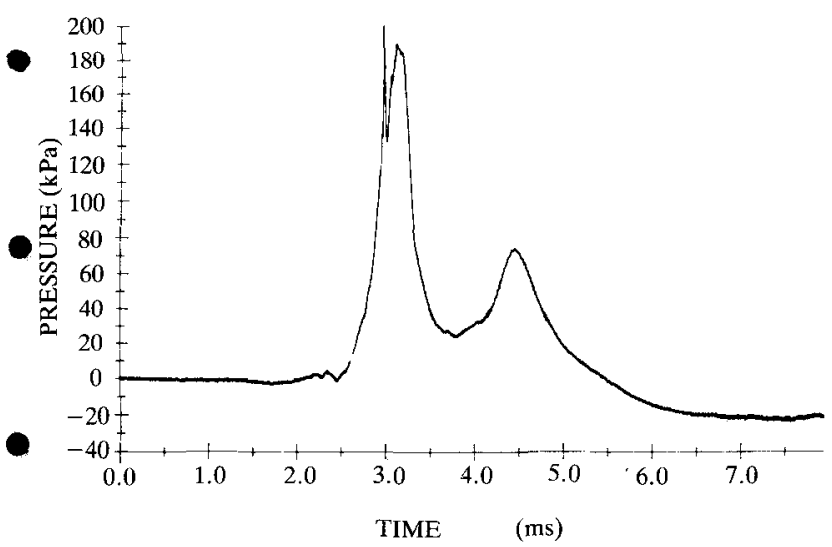

Fig 3. A stress wave propagated in the chest by right lateral impact. The wave was measured by a transducer in the right main bronchus. velocity NP impacts to the lateral chest, contusion may also be seen in the medial border of the lung, a perfect niche for the reflection and reinforcement of the stress waves to produce 'stress concentration'. A locus of $\varrho$ haemorrhagic contamination at hilar and peribronchial sites following blast exposure may result from stress concentration at these structures.

The stress wave is produced by the motion of the body $\overrightarrow{\vec{s}}$ wall but the important feature is the peak velocity that $\overrightarrow{0}$ the body wall attains - this determines the magnitude of $\overline{0}$ the stress wave generated. The magnitude of the gross $\frac{\bar{m}}{\bar{T}}$ distortion in not important. The peak velocity is $\mathbb{Q}$ generally reached within fractions of milliseconds (Fig o 5 ) and so the wave is generated and has traversed the $\infty$ lung well before the peak gross distortion is reached. $\vec{O}$ (Fig 4) (A useful analogy is to strike a cushion hard - the $\overrightarrow{\vec{H}}$ 'boom' is the acoustic wave generated by the initial contact; the distortion of the cushion is a secondary, slower phenomenon).

The relative importance of the high-frequency stress waves and the low-frequency gross distortion in the production of blast lung (and bowel) is the subject of debate currently. This may, at first glance, seem a rather academic argument, but it becomes very important if you are trying to develop, for example, means of providing additional protection to the wearers $\vec{\Phi}-$ Explosive Ordnance Disposal (EOD) suits from th $\overline{\mathrm{T}} \subseteq$ effects of blast waves on the chest. To limit gros thoracic distortions, one approach may be to increase ${ }^{\infty}$

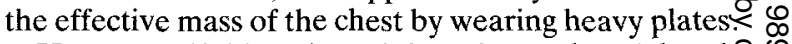

However, if blast lung injury is produced by th 8. propagation of high frequency stress waves, then ratho more subtle approaches are required to attempt tô. 'decouple' or reflect away the wave. Disasters coula occur - covering the chest with foam rubber in an attempt to 'absorb' the energy of a blast wave will $\stackrel{\mathbb{Q}}{\complement}$ actually result in a marked increase in the severity of the $\overrightarrow{\vec{B}}$ internal injury ${ }^{5,6}$. This is probably due to 'acoustic $\frac{3}{3}$ coupling' - under normal circumstances, when a blast wave hits the thorax, a large portion of the energy is reflected away due to the large difference in acoustic properties between air and tissue - the small proportion that is transmitted may have the capacity to injure. By putting a material such as foam rubber with acoustic properties between that of air and tissue, a larger proportion of the incoming blast may be transferred across the foam into the body - the foam acts as a bridge or 'coupler'. Fortunately, there are materials that may overcome this phenomenon by reflecting the blast even more efficiently than the body wall and so significantly reduce the incidence of blast lung 5 .

The production of blast contusions by propagated stress waves is demonstrated in Figure 6. This figure shows a transverse section through the lungs of a rat $\mathrm{N}$ subjected to blast loading on the right lateral thorax. The contusions are not sub-pleural on the lateral border adjacent to the thoracic wall that underwent severe, $\mathbb{\omega}$ gross distortion; they are close to the interface with the 


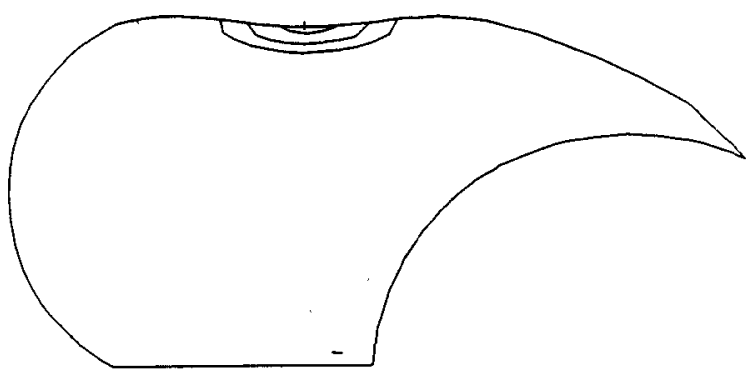

Note to Fig 4:

i. the stress wave is generated in the early stages of the impact when the body wall velocity is high.

ii. the wave precedes the gross distortion of the lung parenchyma.

iii. reflection from the distal margins of the lung leads to re-inforcement with peaks of pressure in the lung that may exceed those generated at the contact surface.
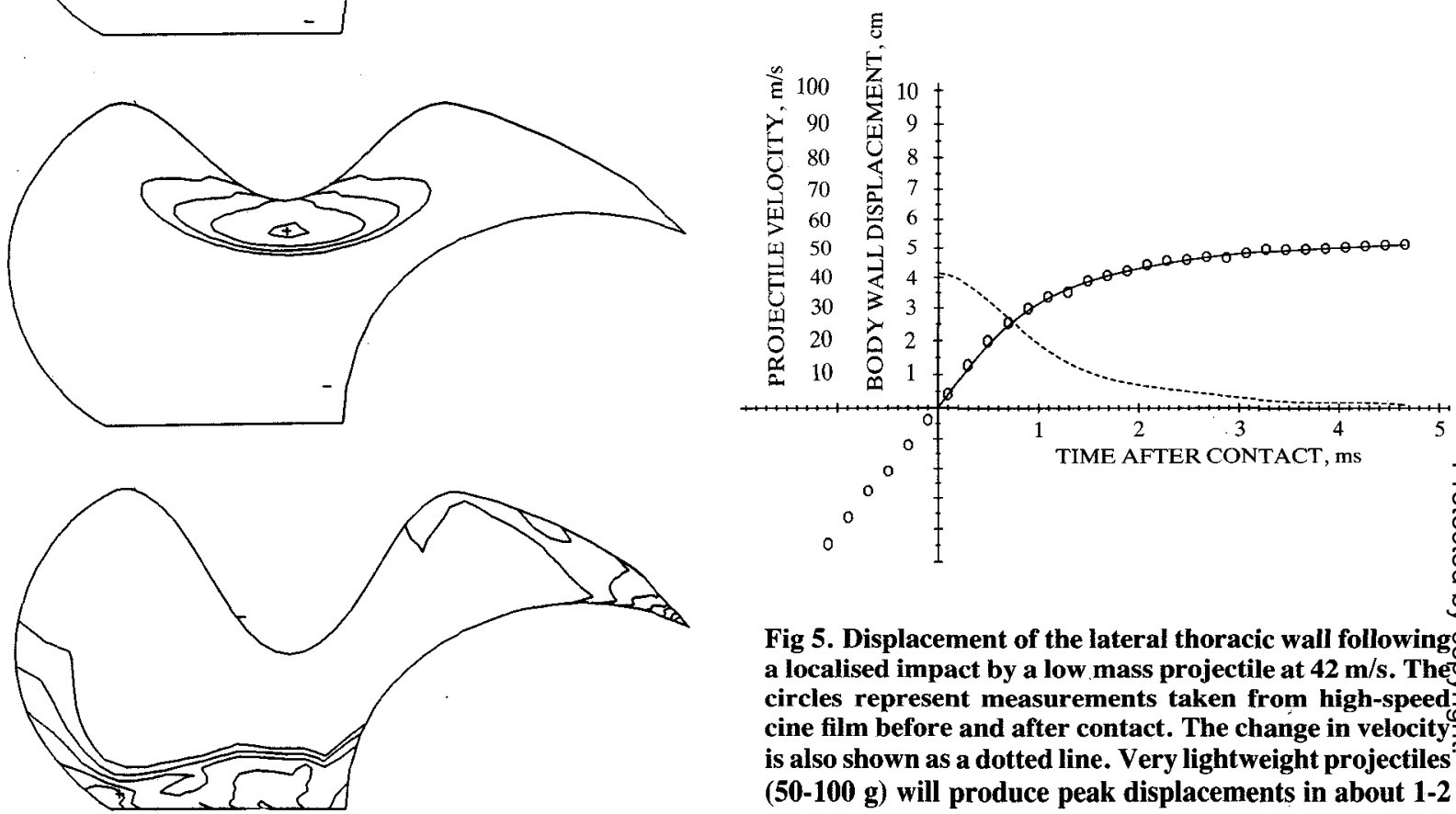

Fig 5. Displacement of the lateral thoracic wall following $\varnothing$ a localised impact by a low mass projectile at $42 \mathrm{~m} / \mathrm{s}$. The circles represent measurements taken from high-speed cine film before and after contact. The change in velocity is also shown as a dotted line. Very lightweight projectiles (50-100 g) will produce peak displacements in about 1-2 ms. Heavy projectiles and the type of high-momentum impacts experienced in car accidents have durations of

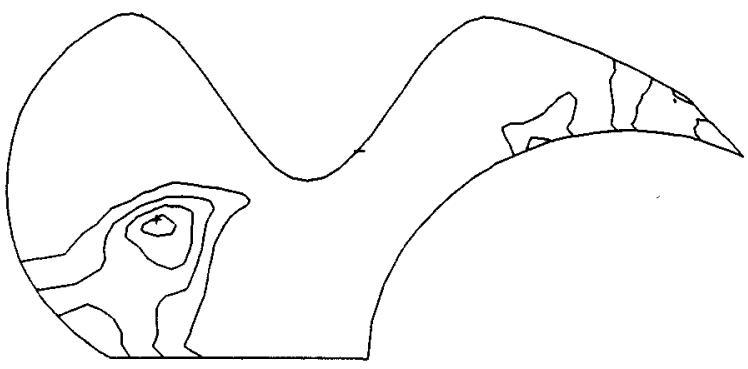

Fig 4. A simulation of the generation and propagation of a stress wave in a transverse section of lung parenchyma following lateral impact upon the body wall by a $69 \mathrm{~g}$ nonpenetrating projectile at $50 \mathrm{~m} / \mathrm{s}$. The stress wave is presented as contours of pressure. The lateral wall is at the top of each figure, mediastinum at the bottom and anterior to the right. Time intervals are $0.5,1.0,2.0$ and 2.5 milliseconds after contact.

mediastinum where reflection and reinforcement occurred. Compare the location of injury in this lung to the pressure contours of Figure 4.

Injuries presumed to result from stress waves may also be seen in bowel - we have seen contusions, using a pig model, in small bowel not only adjacent to the abdominal impact site, but also some distance away (indirect injury). Contusions frequently occur well away from points of fixation of the bowel and are unlikely to have been produced by shear waves. They are often associated with air-filled segments (Fig 7) and under these conditions, the contusions may have three aetiologies: i) stress wave interaction resulting in compression and subsequent re-expansion of the already stressed wall, ii) production of a pressure differential across the bowel wall or iii) 'spalling', the release of energy as the stress wave attempts to propagate from the wall of the bowel into the air filled 


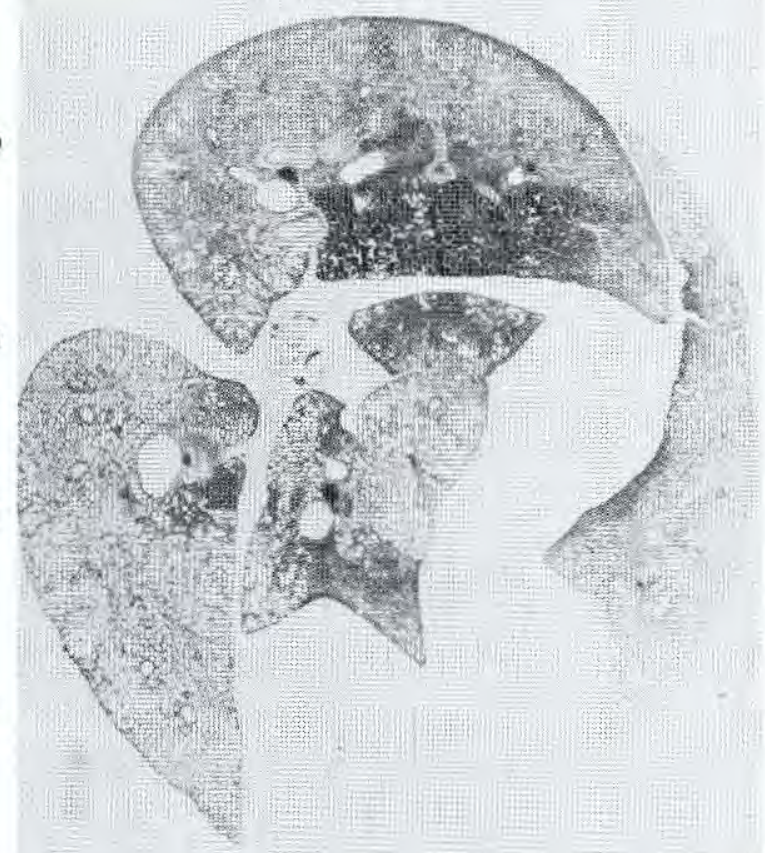

Fig 6. A transverse section of the lungs of a rat subjected to blast overpressure upon the right lateral thorax. The right lung is at the top of the picture, anterior is to the right. The blast wave approached from the top of the picture. The mediastinum and body wall have been removed. Blast contusions are evident close to the distal border of the lung where reflection of stress waves from the mediastinum occurred resulting in stress concentration in this region. Note the absence of injury in the parenchyma immediately beneath the body wall where gross distortions occurred.

lumen. The compressive wave is reflected back at the interface as a tension wave and may injure tissue - most materials are weaker in tension than compression. Spalling is a difficult concept - it is the same phenomenon that sprays droplets of water into the air following an underwater explosion or why particles of rust may be ejected from one side of a metal plate when the other side is struck hard.

Shock waves are fast rising waves of high pressure propagated through the underlying tissue at a velocity faster than the velocity of sound in the tissue. A shock wave can be considered to be a special form of a compression wave and may be produced when the advancing body wall accelerates 'particles' of tissue to a velocity greater than the velocity of sound in the medium. For most body tissues, the velocity of particles

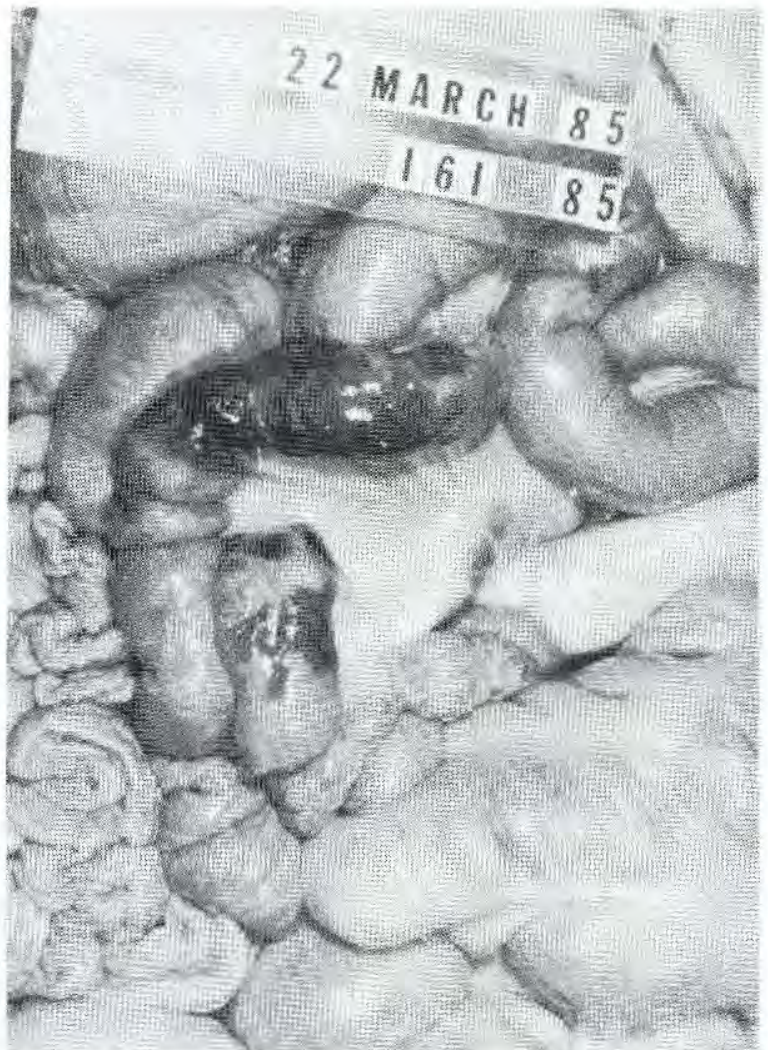

Fig 7. Contusion in an air filled segment of bowel following a high velocity, low displacement impact to the anterior abdominal wall of an anesthetised pig.

accelerated by an impact will never approach the velocity of sound in the tissue (for most soft tissues around $1300-1500 \mathrm{~m} / \mathrm{s} ; 3500 \mathrm{~m} / \mathrm{s}$ for bone). The velocity of sound in lung is around only $15-40 \mathrm{~m} / \mathrm{s}$ and therefore for many impacts, particularly of small lightweight projectiles, the initial body wall velocity may actually exceed the velocity of sound and true shock waves may be propagated initially.

Blast waves provide an extreme example of very fast rates of energy transfer to the body but the impact by NP projectiles may produce equivalent rates of transfer. The duration of an impact is predominantly dependent on the mass of projectile; lightweight projectiles produce impacts of very short duration and high velocity but the actual distortion of the body wall is usually not severe (distortion is dependent upon the momentum (mass $x$ velocity) and in the real world, lightweight, fast projectiles that do not perforate the body wall have a "low" momentum) $)^{3.7}$. Of course, lightweight projectiles that have a capacity for producing NP impact injury are invariably travelling fast and the high initial body wall velocities will tend to excite the high-frequency 
compression waves to produce direct and indirect contusions.

Shear waves may produce marked distortions of internal organs. The distortion produced in organs adjacent to the body wall may produce local shear to result in contusion or even, in severe cases, laceration. Contusion of the right ventricle of the heart following anterior chest impact or laceration of the capsule of the liver and underlying parenchyma are local, direct conscquences of the gross motion of the body wall (Fig 8).

The motion of internai structures occasioned by the displacement of the body wall may be propagated into the body as a shear wave - a transverse wave of long duration and low velocity. The internal disturbances may produce indirect injury resulting from

- asynchronous motion of adjacent, connected structures

- stretching (strain) at sites of attachment

- collision of viscera with stiff structures

The principal features of the motion of the body wall that may determine the incidence of shear-dominated injuries is the maximum displacement of the body wall and the time taken to attain this displacement (Fig 5). The former is governed principally by the momentum of the impact and the latter by the mass of the impactor. Civilian road traffic accidents generally involve large momentum exchange and so the shear (and crush) type injuries tend to predominate under these circumstances.

Examples of injuries produced by strain at sites of attachment are injury to mesentery, laceration of the pedicle of the spleen, aortic rupture (discussed below) and indirect liver tears adjacent to the falciform ligament. Asynchronous motion of attached viscera may be evident as tears of the insertion of the gall bladder to the liver and hacmorrhage into the connective tissue binding the coils of the colon in the pig or serosal haemorrhages in the transverse colon of the human.

The pig is an appropriate model for many aspects of thoracic and abdominal biomechanics, but its spleen is quite unlike that of the human, being a long strap-like organ that traverses the costal margin - the spleen is easily lacerated as an indirect injury in this model, resulting from collision of the body of the spleen with the costal margins, the third type of shear wave induced injury outlined above. An example of this mode of injury in the human would be impact of the body of the pancreas upon the lumbar vertebral bodies.

Although it may be convenient to categorise the types of strain induced by impact, many impacts invoke all categories - local shear, differing motion of adjacent structures and strain at sites of fixation. Impact overlying the heart is a good example and can serve to demonstrate these phemomena. With appropriate instrumentation, these disturbances may be assessed quantitatively and may provide insight on the aetiology of both direct and indirect cardiac contusions and indirect aortic ruptures.

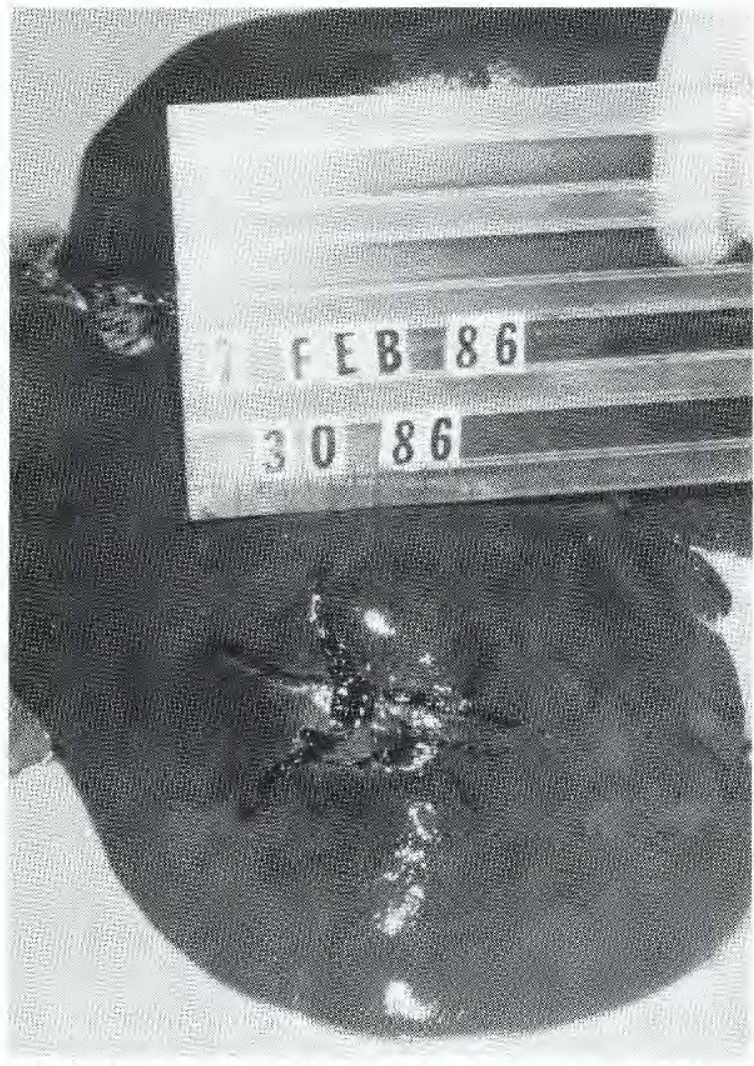

Fig 8. Stellate laceration of the liver - an example of localised direct shear injury.

The heart is a relatively unfixed organ of high inertia the pericardium has loose attachments to the overlying stcrnum and to the sternal part of the diaphragm. The insertions of the great vessels and their associated fibrous connective tissue provide fixation cranially. If the heart is modelled simply as a liquid filled balloon tethered and hanging from a fixed point, the response of the model to a sharp blow would be fairly obvious. During the short duration of the blow, the heavy balloon would remain largely stationary, but locally, the balloon wall would be distorted (local shear). A short time later, the inertia of the balloon as a whole would be overcome and the transfer of momentum would result in the gross motion of the whole balloon away from the impact point with stress of the string suspending it. This mirrors the behaviour of the heart following NP impact to the sternum and is the aetiology of direct and indirect injury to the heart and great vessels..

High speed cine-radiography and flash radiography has been used to measure the distortion and displacement of the heart and aorta during short duration impacts to the mid-sternum of the anaesthetised $\mathrm{pig}^{8}$. Small silver spheres were attached to the heart 
and aorta of pigs at specific anatomical sites whose motion was considered to provide insight on the strains induced under dynamic loadings. Four to five weeks later the pigs were anaesthetised and subjected to impacts to the anterior chest by $3.7 \mathrm{~cm}$ diameter, $0.14 \mathrm{~kg}$ free-flying projectiles at velocities within the range 30-64 $\mathrm{m} / \mathrm{s}$. High speed cineradiographic techniques were used to determine the 2-dimensional motion of the individual spheres at $1 \mathrm{~ms}$ intervals during the impact additionally, 3-dimensional reconstruction was also possible at two defined time intervals during the impact using flash radiography. The distortions of the heart during the impact and the motion of the heart as a whole and the aorta within the thoracic cage could be determined at millisecond intervals.

Figure 9 identifies a number of these spheres on a lateral radiograph of the thorax and upper abdomen of a pig. The percentage change in separation of some of these markers is shown in Figure 10. The peak distortion of the anterior abdominal body wall was reached within 2-3 ms for the particular projectiles used. Within about 4 $\mathrm{ms}$ after contact of the projectile, the heart had been maximally compressed to about $50 \%$ of its preimpact antero-posterior dimension demonstrated as a reduction in the separation of the ANT and POST spheres. This direct cardiac compression resulted in high internal pressures within the cardiac chambers - heart and cardiac contusion may be produced by transfer of this pressure wave down the coronary vessels (contusions are often associated with the interventricular groove) and more probably by shearing stresses induced in the myocardium by the distortion. Direct contact of the sternum upon the heart may itself induce injury but cannot account for contusion on the posterior cardiac surface - the shearing consequent to the ventricular distortion is the likely cause.

Inspection of the separation of the POST and SP markers (Figs 8 and 9) shows that as the heart gradually regains its original anteroposterior dimension, it moves posteriorly towards the spine reaching its peak displacement at about $+15 \mathrm{~ms}$. The heart is also pushed caudally by the angled sternum and these combined gross displacements induce considerable strain on the attachments of the great vessels - laceration of these vessels may be produced if the impacts are very severe.

The aorta is stretched; the SP marker on the 'fixed' aorta and the AOR marker on the 'mobile' arch move apart (Fig 10). Longitudinal tension on the aorta is the probable aetiology of the commonly described aortic rupture at the isthmus just distal to the origin of the left subclavian artery ${ }^{9.10}$. Tears tend to be circumferential rather than longitudinal and this supports the heart/ aorta displacement theory. An alternative theory of an internal bursting force produced by elevated aortic pressure would result in longitudinal tears.

Crush injury is not rate dependent but simply a consequence of applying high, effectively static load to the tissue, for example, retroperitoneal haematoma and bowel laceration seen following gross compression of the anterior abdominal wall resulting in contact with the retroperitoncal surface.

\section{Injury to ribs}

It is obvious to state that direct fractures may occur with loadings on ribs. The likelihood of fracture is principally dependent upon the displacement and the time duration of the impact. Indirect fractures may also occur in ribs and perhaps the most common is fracture

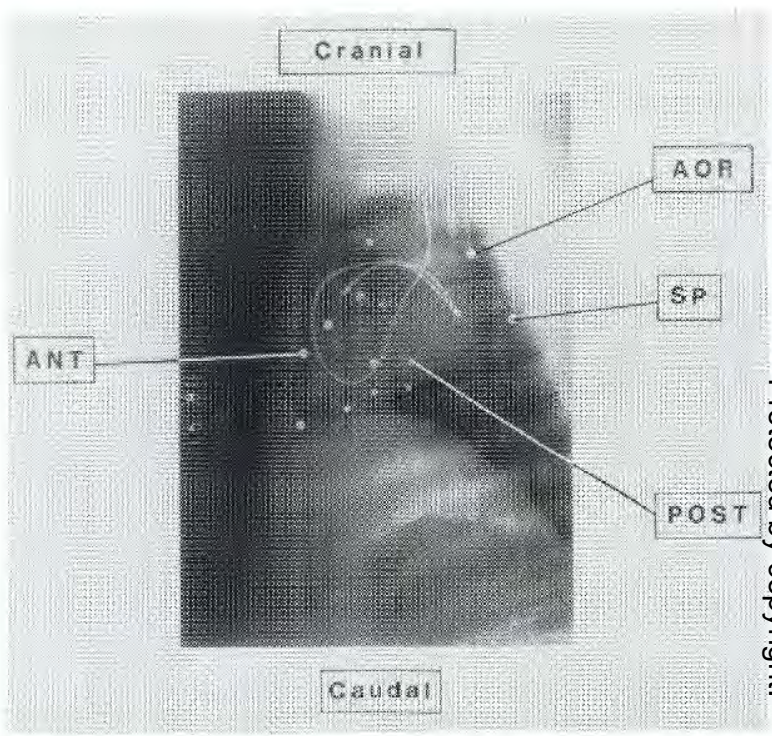

Fig 9. A lateral radiograph of the chest of an anaesthetised pig prior to mid-sternal impact. The silver spheres were attached at previous operation to the heart and aorta; displacement of these markers was tracked by high-speed cine radiography. A pressure transducer is coiled within the right ventricle. Four of the balls are highlighted:

ANT (anterior surface of the heart) was attached to the epicardium to the left of the anterior descending coronary artery.

POST (posterior surface) was attached to origin of the right posterior descending coronary artery from the right circumflex artery.

SP (spine) was adjacent to the lateral border of the thoracic aorta at $\mathrm{T} 6$ level. The aorta is relatively fixed at this level.

AOR (aorta) was attached at the distal angle of the origin of the left subclavian artery from the aortic arch. The aortic is relatively mobile at this level. 


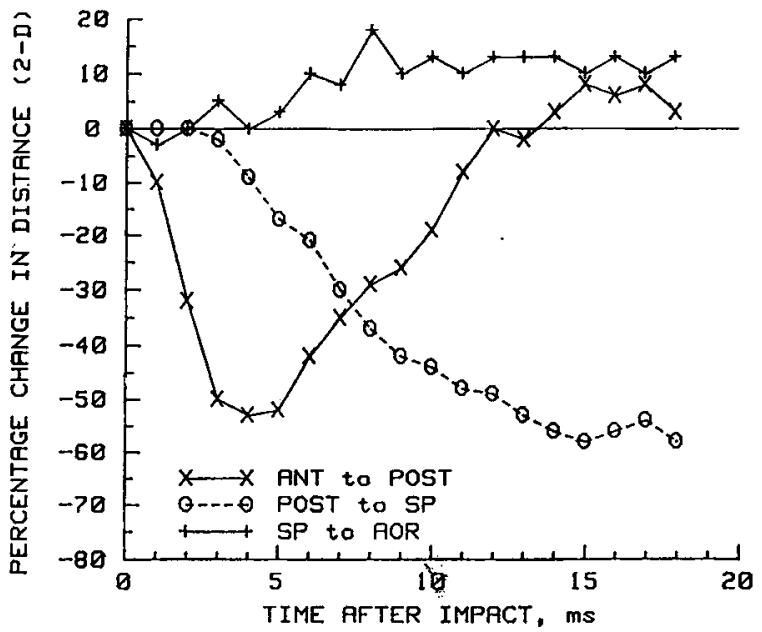

Fig 10. Motion in two dimensions (cranial/caudal and anterior/posterior) of the markers highlighted in Figure 9. Following impact to the anterior chest by a low mass free-flying projectile, peak chest wall displacement was reached in 3 milliseconds. An increase in separation is a positive number on the $Y$ axis. A description of the response is presented in the text.

posterolaterally near the angle of the rib. It can be shown using finite element modelling and beam theory ${ }^{7}$, that with loading to the anterior end of a rib, there are two zones of secondary maximum stress concentration, one laterally and the other posterolaterally at the angle, the sites of indirect fractures. These stress concentrations result from the posterior curvature and caudal angulation of the ribs in the human. Lateral impacts may also produce stress concentration distant from the contact point but the magnitude of the stress is not as great as that produced by equivalent anterior impacts.

In summary, although it may be convenient to ascribe the injuries produced in the torso following impact simply in terms of 'crush', this is an oversimplification and cannot explain many of the characteristic patterns of injury, particularly indirect injury. Energy must be transferred from the body wall to internal sites and this is achieved by the propagation of waves. With increasing body wall velocity the aetiology changes from a sheardominated direct and indirect mechanism producing gross visceral displacements and distortions, to include the involvement of stress or even, under special circumstances, shock waves.

The majority of impact injuries to the torso encountered in civilian medical practice (such as those resulting from road traffic accidents) are produced by shear because they are long duration, 'low' velocity, high momentum impacts producing severe but 'slow' distortions of the body wall. Body wall velocities in the occupants of vehicles subjected to side impacts are less than $15 \mathrm{~m} / \mathrm{s}$ (33 mph).

Most impacts at 'high' velocity (say $>30 \mathrm{~m} / \mathrm{s}$ ) are with projectiles of low mass resulting in short duration, small displacement of the body wall at high velocity. Under these circumstances, stress waves may contribute significantly to the injury mechanism.

It is difficult to apply conventional engineering stress analysis to the human tissues principally because of their poorly defined mechanical properties and the complex interactions between adjacent structures. However, by pursuing appropriate parallel studies on i) the fundamental biomechanical properties of tissue, both human and experimental animal, ii) appropriate modelling using established engineering techniques such as stress analysis and finite element modelling (Fig $4)^{4,7,11}$ and iii) impacts upon anaesthetised animals ${ }^{3,8}$, extrapolations may be made to the human to allow development of protective measures and appropriate injury assessment criteria to reduce the incidence and severity of impact injury to the torso in both civilian and military spheres.

\section{Acknowledgements}

We would like to thank Frazer-Nash (Consultancy) Ltd, Leatherhead, for permission to use the modelling shown in Figure 4. Mr S Challener, Mr I R Bush of Frazer-Nash (Consultancy) Ltd and Mr M G Langdon of the Transport and Road Research Laboratories, Crowthorne provided enlightening discussions on the engineering aspects of impact injury.

\section{REFERENCES}

1. MoRgAGNI J B, Epistola 1761; 54: 140-142.

2. LANGDON M G. Requirements for minimising thoracic injury in side impact experiments. Proc International Research Committee on the Biokinetics of Impacts (IRCOBI). Zurich, 1986; 319-330.

3. COOPER G J and MAYNard R L. An experimental investigation of the biokinetic principles governing nonpenetrating impact to the chest and the influence of the rate of body wall distortion upon the severity of lung injury. Proc IRCOBI Conference on the Biokinetics of Impacts, Zurich, 1986; 331-342.

4. Bush I and Challener I S. Finite element modelling of non-penetrating thoracic impact. Proc IRCOBI Conference on the Biokinetics of Impacts. Sept 1988; Bergisch Gladbach, FRG. (in press).

5. COOPER G J. et al. Blast loading to the thoracic wall - the influence of foam and other materials upon the severity of blast lung injury. $C D E T P 494,1988$.

6. Clemedson C J, et al. Effects of extra- and intrathoracic pressure pattern and lung injuries of rigid and soft protection of thorax in blast exposed rabbits. Forsvarmedicin, 1971; 7: 172-190. 
7. TAYLOR D E M, et al. Stress/strain characteristics of flat bones in relation to non-penetrating injuries of the chest and head. In Material Properties and Stress Analysis in Biomechanics. Ed A L Yettram, Manchester University Press, 1988 (in press).

8. CoOper G J. et al. Cardiovascular distortion in experimental nonpenetrating chest impacts. $J$ Trauma 1984; 24: 188-200.

9. Viano D C. Traumatic aortic ruptures. General Motors
Research Publication GMR-2717, 1978, GM Research Laboratories, Warren Michigan.

10. SEvITT $S$. The mechanism of traumatic rupture of the thoracic aorta BrJ Surg 1977; 64:166-173.

11. TAYLOR D E M. et al. Modelling of the ribs and thoracic cage in studies of the biomechanics of nonpenetrating cheso injury. Proc United Kingdom Simulation Councip Conference on Computer Simulation, Ed R N Zobel;: Bangor, September 1987. 